\title{
Inherited Autoinflammatory Disease with Immunodeficiency Combined with IgA Nephropathy
}

\section{Hereditäre Autoinflammation mit Immunschwäche in Verbindung mit IgA-Nephropathie}

Authors

Zhifeng Jiang, Aiqiao Feng, L I Tao

\author{
Affiliations \\ Nephrology, Xiaogan Hospital Affiliated to Wuhan University \\ of Science and Technology, Xiaogan, China \\ Key words \\ fever, autoinflammatory, immunodeficiency, PLCG2, IgA \\ nephropathy \\ Schlüsselwörter \\ Fieber, Immunschwäche, PLCG2, IgA-Nephropathie, \\ autoinflammatorisch \\ Published online $\quad 14.05 .2020$ \\ Bibliography \\ Akt Rheumatol 2020; 45: 574-576 \\ DOI 10.1055/a-1135-8602 \\ ISSN 0341-051X \\ (c) 2020. Thieme. All rights reserved. \\ Georg Thieme Verlag KG, Rüdigerstraße 14, \\ 70469 Stuttgart, Germany \\ Correspondence \\ Dr. Zhifeng Jiang \\ Nephrology \\ Xiaogan Hospital Affiliated to Wuhan University \\ of Science and Technology \\ No. 6, Square Street, Xiaogan City \\ 432100 Xiaogan \\ China \\ Tel.: + 13995893375, Fax: + 13995893375 \\ xjiang292@sina.com
}

\section{ABSTRACT}

The etiology of unexplained periodic fever is often complex, and hereditary factors play an important role. This article describes a 26-year-old chinese women with intermittent fever for 9 years, with 10-year history of IgA nephropathy. Her fever is relieved during pregnancy, but after a baby is born, fever reappears, accompanied by headache, gasping after activity, chest pain, abdominal pain, blood in the stool, ataxia, intermittent back erythema, skin biopsy suggests amyloidosis, the autoinflammatory PLCG2 associated antibody deficiency and immune dysregulation was diagnosed by genetic testing. The fever was gradually relieved after treatment with rilonacept.

\section{ZUSAMMENFASSUNG}

Die Ätiologie des ungeklärten periodischen Fiebers ist häufig komplex. Erbliche Faktoren spielen eine bedeutende Rolle. Dieser Artikel beschreibt eine 26-jährige chinesische Patientin mit intermittierendem Fieber seit 9 Jahren und 10-jähriger Vorgeschichte einer IgA-Nephropathie. Während ihrer Schwangerschaft war das Fieber rückläufig, doch nach der Geburt des Kindes trat es wieder auf, begleitet von Kopfschmerzen, keuchender Atmung nach Aktivität, Brustschmerzen, Bauchschmerzen, Blut im Stuhl, Ataxie und intermittierendem Erythem am Rücken. Eine Hautbiopsie deutete auf eine Amyloidose hin, während durch Gentests ein autoinflammatorischer PLCG2-assoziierter Antikörpermangel und eine Immunschwäche diagnostiziert wurden. Das Fieber ging nach Behandlung mit Rilonacept allmählich zurück.

\section{Background}

Autoinflammatory diseases are inherited immunological disorders caused by aberrant activation of innate immune cells, primarily neutrophils, and macrophages. Pathogenic inflammation of autoinflammatory diseases is attributed to the abnormal activation of the non-antigen-dependent immune system. Several diseases manifested as recurrent fever, and are known as periodic fever syndromes; these might also be dominated by other clinical features.

Autoinflammatory syndromes include monogenic and polygenic diseases that manifest as lifelong recurrent systemic inflamma-

tion with organ-specific involvement, usually affecting the serosa, skin, joints, bones, gastrointestinal tract, and the central nervous system. The most well-characterized autoinflammatory diseases known to be caused by a single gene mutation.

\section{Case report}

A 26-year-old Chinese woman was diagnosed with IgA nephropathy by renal biopsy 11 years ago due to edema and hematuria, but no obvious relief from proteinuria was experienced with glucocorticoid. After 1 year, renal biopsy was repeated in another hospital, 
which also suggested IgA nephropathy with focal crescent formation. After glucocorticoid and cyclophosphamide pulse treatment, proteinuria was relieved gradually. Fever was detected repeatedly from November 2012 to February 2018, and each time, it lasted for several days. The normal features included procalcitonin, blood culture, erythrocyte sedimentation rate, C-reactive protein, disruption of liver and kidney function, electrolytes, fungal examination, anti-neutrophil cytoplasmic antibody, autoantibodies lupus antibodies, tumor markers, serum protein electrophoresis, immunity fixed electrophoresis, endocrine hormones, and no abnormalities in the head, chest, and abdomen CT. In addition, there were no positive findings such as bone marrow puncture and lumbar puncture as assessed by bone marrow flow cytology. No significant relief was experienced by antibiotics, glucocorticoid, immunosuppression, and diagnostic anti-tuberculosis treatment, and five years ago, the skin of the forearm was found to be red and itchy. From 2016 to 2017, the patient stopped using glucocorticoids and immunosuppressants after remission, had a successful pregnancy, and gave birth to a healthy boy at term. During pregnancy, the patient's fever disappeared spontaneously, and no drugs, including antipyretics, were applied.

In June 2018, the fever recurred, and after the activity, gasping, intermittent hemoptysis, abdominal pain, chest pain, hematuria, intermittent hypertension/hypotension $(180 / 110 \mathrm{mmHg}$, $80 / 50 \mathrm{mmHg}$, respectively), and often fall, electrocardiogram showed frequent ventricular premature beats. However, the T cell subsets were normal , IL-6: $4.15 \mathrm{pg} / \mathrm{mL}$ (reference: $0.1-2.9$ ) , and virus test negative. Chest CT indicated pulmonary interstitial changes and pulmonary granuloma. No obvious positive findings were observed by gastroscopy, Congo red staining was negative in the gastric mucosa, and no positive findings were detected in bronchoscopy, and Congo red staining of 10-year-old renal tissue samples showed negative results. Previously, the patient had knee pain in early childhood and intermittent headache for 12 years. One year ago, the MR of the knee showed an abnormal signal of the right femur and tibia, considering metabolic changes, softening of the tibial cartilage, and a small amount of effusion in the supraorbital sac and joint cavity. No relief was experienced with glucocorticoids and antibiotics. In July 2018, the whole exon gene test revealed a mutation in the PLCG2 gene, chr16:81902844, c.505A > G.p.lle 169Val ( Fig. 1), while back skin biopsy suggested amyloidosis ( $>$ Fig. 2. HE staining suggests amyloid deposition in the subcutaneous papilla, $4 \times 10$-fold). The patient's father was heterozygous, and the mother had no abnormalities at that site. The family had no history of similar diseases.

According to the patient's medical history, the final diagnosis was autoinflammatory PLCG2-associated antibody deficiency and immune dysregulation (APLAID). Hence, rilonacept (160 mg qd) was administered for 2 weeks; the fever was relieved gradually, and no adverse and unanticipated events were observed at the follow-up.

\section{Discussion}

Genetically defined, autosomal dominant human primary immunodeficiencies have been found to manifest in various combinations of phenotypes belonging to five broad categories: autoimmunity, autoinflammation, allergy, infection, and malignancy [1]. PLCG2-rela-

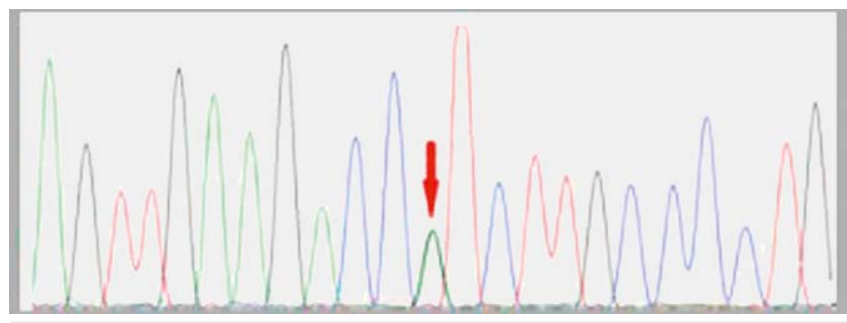

- Fig. 1 The whole exon gene test revealed a mutation in the PLCG2 gene, chr16:81902844, c.505A>G.p.lle169Val.

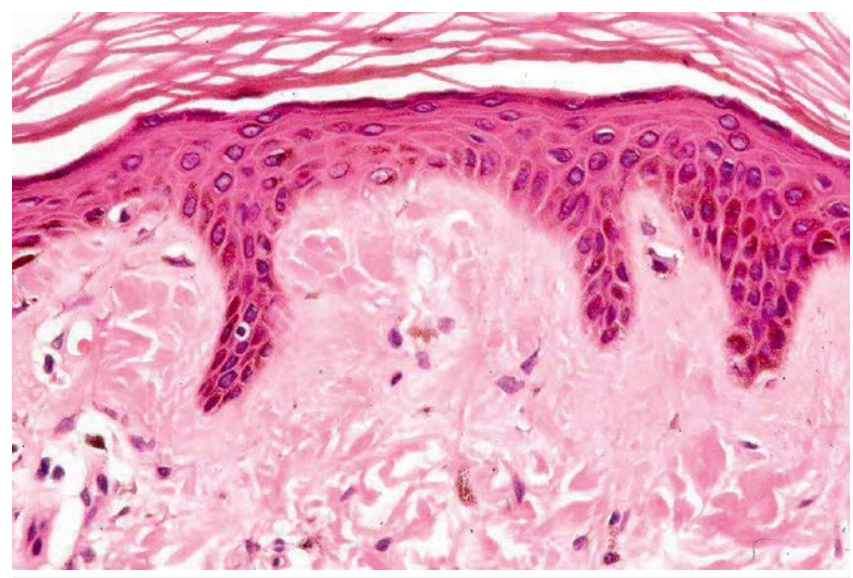

- Fig. 2 Back skin biopsy suggested amyloid deposition in the subcutaneous papilla (HE staining $4 \times 10$-fold).

ted immunodeficiencies are associated with the first four, and there is no evidence of an association with malignancies [2].

APLAID is an autoinflammatory disease, with PLCG2 gene mutation as the characteristic Change [1]. PLCG2 encodes phospholipase Cy2 (PLC gamma 2), an enzyme with key regulatory roles in various immune and inflammatory pathways [1]. Recent evidence suggested that PLCG2 mutations are involved in several human pathologies. Deletions of exons 19 or 20-22 cause cold urticaria and PLCY2-associated antibody deficiency and immune dysregulation (PLAID) [3, 4], while a point mutation (S707Y) is the basis of autoinflammation and PLCY 2-associated antibody deficiency as well as immune dysregulation (APLAID) [3].

Interestingly, the patient did not use any medication during pregnancy, the disease ceased to appear, no fever and other related discomforts were noted, and a baby boy was was born successfully. Reportedly, familial Mediterranean fever and Periodic fever aphthousstomatitis pharyngitis cervical-adenitis syndrome (PFAPA) patients may experience remission after pregnancy, which could be attributed to changes in hormones during pregnancy [5]. It is speculated that PFAPA syndrome is a Th1-type immune disease that may be improved by Th1 to Th2 shift caused by pregnancy [6]. Presently, there is no report of APLAID combined with pregnancy, and the correlation between self-inflammation and pregnancy should be investigated.

Some patients with periodic fever can be combined with amyloidosis, Muckle-Wells syndrome (MWS), and familial Mediterranean fever patients can be complicated by amyloidosis, proteinuria, and renal failure $[7,8]$; however, APLAID/PLAID complicated with 
amyloidosis has not been reported. In this case, patients with gastrointestinal bleeding and intermittent chest pain showed arrhythmia on the electrocardiogram, while blood pressure fluctuations considered disruptions in the digestive tract, myocardium, and amyloidosis of the heart conduction system .

APLAID combined with IgA nephropathy has not yet been reported. Previous studies have shown that IL-1 $\beta$ is involved in the pathogenesis of spontaneous and passively induced IgA nephropathy models, in which the activation of NLRP3 inflammasome contributes to the pathogenesis of IgA nephropathy [9]. In addition, Robinet et al. reported a case of Muckle-Wells syndrome with $\lg A$ nephropathy and renal amyloidosis, both fever and proteinuria were significantly reduced by using interleukin-1 blocker [10]. We hypothesized that over-activation of IL-1 may be involved in the pathogenesis of both APLAID and IgA nephropathy.

Autoinflammatory diseases manifest multiple features of the central nervous system, including chronic aseptic meningitis and brain atrophy, sensorineural hearing loss, early-onset hemorrhagic and ischemic strokes, mental retardation, cerebellitis, ataxia, and severe encephalopathy with brain calcifications [11]. However, APLAID combined with central nervous system performance has not yet been reported. In this case, the patient had repeated ataxia, headache, nausea, and vomiting, but no obvious abnormalities in the head MR. Thus, the specific mechanism is still unclear.

Most autoinflammatory diseases are associated with the overproduction of IL-1 and IL-6 [10]. With the introduction of anti-IL-1 drugs, the treatment of such diseases has become effective. Currently, three commercially available anti-IL-1 drugs are anakinin, rilonacept, and canakinumab [13]. Some patients with cryopyrin- associated periodic syndromes (CAPS), such as IL-1 blockers, are ineffective, and IL-6 blockers can also be tested [13]. Reportedly, IL-1 blockers have achieved significant effects in the treatment of ocular lesions in patients with PLAID [12]. This patient also experienced symptoms relief after intramuscular injection of rilonacept $160 \mathrm{mg}$ weekly.

APLAID complicated with gastrointestinal bleeding and ataxia has not been reported before, and the specific pathophysiological mechanism is currently unclear. Thus, and further laboratory studies are essential with additional cases. Also, no other accompanying symptoms were noted in the early stage of fever. As the disease progressed and repeated, respiratory, digestive, cardiovascular, and other systemic damages appeared eventually, suggesting that APLAID may be poor in the absence of effective treatment.

In summary, APLAID appears in a variety of phenotypes and damage to multiple systems, such as skin, gastrointestinal tract, lungs, cardiovascular, bones, central nervous system, endocrine and immune system, and accompanied with amyloidosis. The specific pathophysiological mechanism of APLAID was complicated with multiple system damage, and hence, the correlation between autoinflammatory diseases and IgA nephropathy as well as pregnancy needs further investigation.

\section{Authors' contributions}

ZFJ was responsible for the diagnosis, treatment, follow-up, writing, and submission of the report. AQF was involved in the diagnosis and treatment of the patient. TL was involved in the diagnosis and treatment of the patients.

\section{Acknowledgments}

Thanks to the patient and all the staff of the nephrology team.

\section{Funding}

There are no competing interests or funding conflict.

\section{Consent for publication}

The figure and clinical information in this article were utilized for verbal consent from the patient.

\section{Competing interest}

The authors declare that they have no competing interests.

\section{References}

[1] Boisson B, Quartier P, Casanova JL. Immunological loss-of-function due to genetic gain-of-function in humans: autosomal dominance of the third kind. Curr Opin Immunol 2015; 32: 90-105

[2] Walliser Claudia, Wist Martin, Hermkes Elisabeth et al. Functional characterization of phospholipase C- $\mathrm{y} 2$ mutant protein causing both somatic ibrutinib resistance and a germline monogenic autoinflammatory disorder. Oncotarget 2018; 9: 34357-34378

[3] Zhou Qing, Lee Geun-Shik, Brady jillian et al. A Hypermorphic Missense Mutation in PLCG2, Encoding Phospholipase Cy2, Causes a Dominantly Inherited Autoinflammatory Disease with Immunodeficiency. Am J Hum Genet 2012; 91: 713-720

[4] Walliser C, Wist M, Hermkes E et al. Functional characterization of phospholipase $\mathrm{C}-\mathrm{\gamma} 2$ mutant protein causing both somatic ibrutinib resistance and a germline monogenic autoinflammatory disorder. Oncotarget 2018; 9: 34357-34378

[5] Kim Kwang Taek, Jang Hyun Joo, Lee Jae Eun. Familial Mediterranean Fever WithComplete Symptomatic Remission During Pregnancy. Intest Res 2015; 13: 287-290

[6] Ota Kuniaki, Kwak-Kim Joanne et al. Pregnancy complicated with PFAPA (periodic fever,aphthous stomatitis, pharyngitis and cervical adenitis) syndrome: a case report. BMC Pregnancy Childbirth 2018; 18: 207

[7] Almeida De Jesus Adriana, Goldbach-Mansky Raphaela. Monogenic Autoinflammatory Diseases: Concept And Clinical Manifestations. Clin Immunol 2013; 147: 155-174

[8] Stella A, Cortellessa F, Scaccianoce G et al. Familial Mediterranean fever: breaking all the (genetic) rules. Rheumatology (Oxford) 2019 Mar 1;58(3): 463-467

[9] Tsai Yu-Ling, Hua Kuo-Feng, Chen Ann et al. NLRP3 inflammasome: Pathogenic role and potential therapeutic target for IgA nephropathy. Sci Rep 2017; 7: 41123

[10] Robinet G, Renaudineau E, Lamaison C et al. Renal amyloidosis revealing a cryopyrin associated periodi c syndrome. Ann Pathol 2018; 38: $401-406$

[11] Uccelli A, Gattorno M. Neurological manifestations in autoinflammatory diseases. Clin Exp Rheumatol 2018; Suppl 110(1): 61-67

[12] Di Zazzo A, Tahvildari M, Florakis G] et al. Ocular Manifestations of Inherited Phospholipase-Cy2-Associated Antibody Deficiency and Immune Dysregulation. Cornea 2016; 35: 1656-1657 